(C) ACM 2018. This is the author's version of the work. It is posted here by permission of ACM for your personal use. Not for redistribution. The definitive version was published in Proceedings of the 8th International Conference on Web Intelligence, Mining and Semantics, ISBN: 978-1-4503-5489-9, DOI: https://doi.org/10.1145/3227609.3227678

\title{
Minimizing Efforts in Reconciling Participatory Sensing Data
}

\author{
Phan Thanh Cong \\ University of Technology \\ Ho Chi Minh, Vietnam \\ ptcongbk@gmail.com \\ Nguyen Quoc Viet Hung \\ Griffith University \\ Gold Coast, Australia \\ quocviethung.nguyen@griffith.edu.au
}

\author{
Nguyen Thanh Toan \\ University of Technology \\ Ho Chi Minh, Vietnam \\ nguyentoanit@gmail.com \\ Bela Stantic \\ Griffith University \\ Gold Coast, Australia \\ b.stantic@griffith.edu.au
}

\begin{abstract}
Participatory sensing has emerged as a new data collection paradigm, in which humans use their own devices (cell phone accelerometers, cameras, etc.) as sensors. This paradigm enables to collect a huge amount of data from the crowd for world-wide applications, without spending cost to buy dedicated sensors. Despite of this benefit, the data collected from human sensors are inherently uncertain due to no quality guarantee from the participants. Moreover, the participatory sensing data are time series that not only exhibit highly irregular dependencies on time, but also vary from sensor to sensor. To overcome these issues, we study in this paper the problem of reconciling probabilistic data from given (uncertain) time series collected by participatory sensors. More precisely, an iterative process is executed in which we exchange between two mutual reinforcing routines: (i) aggregating probabilistic time series from multiple sensors and expert input, (ii) validating them by expert knowledge with minimal effort. Through extensive experimentation, we demonstrate the efficiency and effectiveness of our approach on both real data and synthetic data.
\end{abstract}

\section{CCS CONCEPTS}

- Information systems $\rightarrow$ Sensor networks; Data analytics; Probabilistic retrieval models;

\section{KEYWORDS}

participatory sensing, trust management, probabilistic database

ACM Reference Format:

Phan Thanh Cong, Nguyen Thanh Toan, Nguyen Quoc Viet Hung, and Bela Stantic. 2018. Minimizing Efforts in Reconciling Participatory Sensing Data. In WIMS '18: 8th International Conference on Web Intelligence, Mining and Semantics, fune 25-27, 2018, Novi Sad, Serbia. ACM, New York, NY, USA, 12 pages. https://doi.org/10.1145/3227609.3227678

Permission to make digital or hard copies of all or part of this work for personal or classroom use is granted without fee provided that copies are not made or distributed for profit or commercial advantage and that copies bear this notice and the full citation on the first page. Copyrights for components of this work owned by others than ACM must be honored. Abstracting with credit is permitted. To copy otherwise, or republish, to post on servers or to redistribute to lists, requires prior specific permission and/or a fee. Request permissions from permissions@acm.org.

WIMS '18, fune 25-27, 2018, Novi Sad, Serbia

(C) 2018 Association for Computing Machinery.

ACM ISBN 978-1-4503-5489-9/18/06 .. \$15.00

https://doi.org/10.1145/3227609.3227678

\section{INTRODUCTION}

Participatory sensing, where participants proactively report their observations, has emerged as an important data collection paradigm. In this paradigm, human acts as the sensors or employs their own devices to perform sensing tasks [2, 6, 29, 39, 40, 51, 53, 54, 62]. Examples of these sensors include cell phone accelerometers, cameras, and GPS devices. As the number of sensors owned by an individual increases, participatory sensing is becoming popular. The potential of participatory sensing is tremendous as it can harness the wisdom of the crowd to collect data for wide-ranging applications such as geotagging, environmental monitoring, and public health. These applications would be financially infeasible if traditional approaches using dedicated sensors are applied. Although applications of participatory sensing are abundant, it faces a serious problem from the inferior quality of collected data. Data collected from individual participants and their devices are inherently uncertain due to low sensor quality, unstable communication channels or even intent to cheat the system $[7,8,15,25,38,48,56-58,61]$.

One of the most effective ways to deal with uncertain data is to employ probabilistic approaches. In recent years there have been a plethora of methods for managing uncertain data [3-5, 11, 36, $41,49]$. These methods are typically based on the assumption that probabilistic data is available. One of the most important challenges in managing probabilistic data is how to deal with the data quality. A community of sensors may contain hundreds or thousands of nodes, which make it extremely difficult to handle if we deal with the data from each sensor alone. To make matters worse, the quality of the sensors are highly different. For example, a sensor may suffer from internal problems such as discharged batteries or external problems such as bad weather, which renders it to function improperly. As a result, the quality of the data collected from the sensors varies from sensor to sensor and from time to time [19, 20, 23, 24, 26, 26$28,47,50]$.

To circumvent these crucial problems, we propose a reconciliation process to combine the probabilistic time series from numerous sensors and validate the probabilisitic data using expert knowledge. While user input is incorporated continuously to improve the results of automatic aggregation of multiple sensors, our ultimate goal is to instantiate an accurate participatory data, even if not all data have been validated. By (i) inferring the trustworthinesss of non-validated data from those that have been validated, and by (ii) guiding a user in the validation process, we reduce the amount of manual effort needed to achieve a specific level of result accuracy. 
Both steps, probabilistic aggregation and user guidance, are interrelated. On the one hand, aggregation exploits mutual reinforcing relations between sensors and their readings, which are further justified based on the user input. On the other hand, a user is guided based on the potential effect of the validation of a data reading for probabilistic aggregation. By combining inputs from multiple sensors and expert user, we are able to achieve higher accuracies.

Our contributions and the outline of this paper can be summarized as follows.

- Section 2: We formally discuss the elements and the problem of reconciling participatory data. We also provide an overview of our approach, which requires realization of two components: aggregating probabilistic data and guiding user validation.

- Section 3: We propose an algorithm to effectively measure the trust scores of both the sensors and their data. The trust scores are computed based on the mutually reinforcing relationship between the sensors and the data they provide. Based on the trust scores of the sensors and their data, we discuss a method to combine the probabilistic time series from all sensors.

- Section 4: We design a guiding mechanism to minimise user effort in the reconciliation process, which measures the uncertainty of participatory data and chooses the readings for which manual validation is most beneficial. The faster the uncertainty converges, the less validation effort is spent.

The remaining sections are organized as follows. Section 5 demonstrates experimental results. Section 6 summarizes related works, before Section 7 concludes the paper.

\section{MODEL AND PROBLEM STATEMENT}

In this section, we first describe the elements of our problem. Secondly, we state the problem we want to solve formally. Then, we give an overview of our solution to the problem.

\subsection{Model}

We denote $p \mathcal{D}=\left\{p S_{1}, \ldots, p S_{n}\right\}$ as a set of probabilistic time series where a probabilistic time series $p S_{i}=\left\langle p_{1}\left(R_{1}^{i}\right), \ldots, p_{m}\left(R_{m}^{i}\right)\right\rangle$ constructed from a time series $S_{i}$ is a sequence of probability density functions $p_{j}\left(R_{j}^{i}\right)$. In other words, we can write participatory sensing data as an $n \times m$ density distribution matrix:

$$
p \mathcal{D}=\left(\begin{array}{ccc}
p_{1}\left(R_{1}^{1}\right) & \ldots & p_{m}\left(R_{m}^{1}\right) \\
\ldots & \ldots & \ldots \\
p_{1}\left(R_{1}^{n}\right) & \ldots & p_{m}\left(R_{j}^{n}\right)
\end{array}\right)
$$

Let us denote $\mathcal{R}=\left\{R_{1}, \ldots, R_{m}\right\}$ as the set of sensor reading random variables from timestamp 1 to timestamp $m$. User input is modelled by a probabilistic checking function $e: \mathcal{R} \rightarrow \Omega \cup\{\Theta\}$, where $\Omega$ is the set of all possible probability density functions and the label $\Theta$ denotes that a reading has not yet been validated. It is noteworthy that this model is generic: we can accept user input as a single value $e: \mathcal{R} \rightarrow[0,1]$ (which could be transformed to a density function where the probability of the given value is one) or a value range $e: \mathcal{R} \rightarrow 2^{[0,1]}$ (which could be transformed to a a uniform or normal distribution falled in the given range).
From the set of probabilistic time series $p \mathcal{D}$, we want to determine a grounded probabilistic time series $\mathcal{G}=\left\langle p_{1}\left(G_{1}\right), \ldots, p_{m}\left(G_{m}\right)\right\rangle$. Each probability density function $p_{t}\left(G_{t}\right) \in \mathcal{G}$ is aggregated from the set of probability distributions provided by all sensors and user input at timestamp $t$, which we denote as $\Omega_{t}=\left\{p_{i}\left(R_{i}^{1}\right), \ldots, p_{i}\left(R_{i}^{n}\right)\right.$, $\left.e_{t}\left(R_{t}\right)\right\}$. We provide a summary of notations used in this paper in Table 1.

\subsection{Problem statement}

While our objective is to instantiate a trusted set of time series, our work combines automated probabilistic aggregation with input from users validating sensor readings. However, such validation is costly, in terms of user hiring cost and time. Therefore, user input is commonly limited by an effort budget, which leads to a trade-off between validation accuracy and invested effort.

Going beyond this trade-off, we aim at minimising the user effort invested to reach a given validation goal. We consider data validation as an iterative process with a user validating the value of a reading in each iteration. This process halts either when reaching a validation goal or upon consumption of the available effort budget. The former relates to the desired result quality, e.g., a threshold on the accuracy of the aggregated time series. The latter defines an upper bound for the number of validations by a user and, thus, iterations of the validation process.

Formally, for a participatory sensing data $p \mathcal{D}$, conducting data validation leads to a sequence of aggregated time series $\left\langle\mathcal{G}_{0}, \mathcal{G}_{1}, \ldots\right.$, $\left.\mathcal{G}_{k}\right\rangle$, termed a validation sequence. Each $\mathcal{G}_{i}$ represents the time series obtained after the $i$-th iteration of the validation process. Given an effort budget $b$ and a validation goal $\Delta$, we refer to a sequence $\left\langle\mathcal{G}_{0}, \mathcal{G}_{1}, \ldots, \mathcal{G}_{k}\right\rangle$ as being valid, if $k \leq b$ and $\mathcal{G}_{k}$ satisfies $\Delta$. Let $\mathcal{V}(\Delta, b)$ denote a finite set of valid validation sequences that can be created by instantiations of the validation process. Then, a validation sequence $\left\langle\mathcal{G}_{0}, \mathcal{G}_{1}, \ldots, \mathcal{G}_{k}\right\rangle \in \mathcal{V}(\Delta, b)$ is minimal, if for any validation sequence $\left\langle\mathcal{G}_{0}^{\prime}, \mathcal{G}_{1}^{\prime}, \ldots, \mathcal{G}_{k^{\prime}}^{\prime}\right\rangle \in \mathcal{V}(\Delta, b)$ it holds that $k \leq k^{\prime}$.

Problem 1 (Reconciling Participatory Data). Let $p \mathcal{D}$ be a participatory sensing data and $\mathcal{V}(\Delta, b)$ a set of valid validation sequences for an effort budget $b$ and $a$ goal $\Delta$. The problem of effort minimisation in reconciling participatory data is the identification of a minimal sequence $\left\langle\mathcal{G}_{0}, \mathcal{G}_{1}, \ldots, \mathcal{G}_{k}\right\rangle \in \mathcal{V}(\Delta, b)$.

As detailed above, a validation goal is commonly defined in terms of a threshold on the accuracy of aggregated time series. However, solving Problem 1 is challenging. Sensor readings are not independent, but subject to mutual reinforcing relations with sensors and timestamps. Consequently, the validation of one reading may affect the probabilistic assessment of other readings. Furthermore, sensors with noises influence probabilistic aggregation and may distort the evaluation of a reading's accuracy. Finally, there is a computational challenge and finding an optimal solution to Problem 1 quickly becomes intractable: all permutations of all subsets (of size $\leq b$ ) of readings would have to be explored.

\subsection{Approach Overview}

To address the problem of effort minimisation in reconciling participatory data, we consider a process that guides a user in the 
Table 1: Summary of Notations

\begin{tabular}{cl}
\hline Symbol & Description \\
\hline$R_{t}^{i}$ & Random variable associated with $r_{t}^{i}$ \\
$p_{t}\left(R_{t}^{i}\right)$ & Probability density function of $R_{t}^{i}$ at time $t$. \\
$p \mathcal{D}$ & Participatory sensing data. \\
$p S_{i}$ & A probabilistic time series. \\
$\Omega_{t}$ & A set of probability density functions at time $t$. \\
$p_{t}\left(G_{t}\right)$ & An aggregated probability density function at time $t$ \\
$\mathcal{N}\left(\mu, \sigma^{2}\right)$ & Normal (Gaussian) probability density function \\
& with mean $\mu$ and variance $\sigma^{2}$. \\
$\mathcal{G}$ & The aggregated time series. \\
\hline
\end{tabular}

validation of readings, which continuously updates an aggregated probabilistic time series $\mathcal{G}$. The general idea of the reconciliation process is summarised as follows: user input shall be sought solely on the 'most promising' unverified readings, i.e., those for which manual validation is expected to have the largest impact on the accuracy of aggregated probabilistic time series. Each iteration of the process consists of:

(1) selecting a reading $R$ for which feedback shall be sought;

(2) eliciting user input on the accuracy of $R$, which is represented by the validation function, $e(R)$;

(3) inferring the implications of the user input on the participatory sensing data $p \mathcal{D}$;

(4) deciding on the aggregated time series $\mathcal{G}$ that captures the readings that are assumed to be accurate.

To realise the above process, steps (1), (3), and (4) need to be instantiated with specific methods. An example for a straight-forward instantiation would be a validation process that selects a reading randomly; limits the inference to the reading for which feedback has been sought. In this paper, we present methods for a more elaborated instantiation of the above process that exploit the mutual reinforcing relations between sensors and readings to infer the implications of user input and decide on the aggregated probabilistic time series. Furthermore, we also show how to select the readings for which manual validation is most beneficial.

\subsection{Complete Framework}

Fig. 1 represents an overview of our framework. We start with the time series data collected by participatory sensing. As shown in Fig. 1, the input of our framework is a set of probabilistic time series, where each sensor is represented by a probabilistic time series. More precisely, a probability distribution is used to approximate the expected true value at each timestamp in a probabilistic time series.

In order to leverage the wisdom of the crowd and handle the huge amount of data from numerous sensors, we propose to aggregate the probabilistic time series. To this end, we combine all the probabilistic time series in a Reconciliation procedure, which implements the above iterative process for reconciling participatory data. It involves the exchange between Guiding and Aggregating components. While the former is responsible for the first two steps, the latter realizes the last two steps of the reconciliation process.

Following this general structure, our framework requires the realization of the following components.
Aggregating. Given a set of probabilistic time series generated by the Computing Likelihoods component, the Aggregating component combines all probabilistic time series into one probabilistic time series. Since the sensors are not reliable, in order to aggregate the probabilistic time series, we first need to measure the reliability of the sensors and their data. We achieve this by computing the trust scores of the sensors and their data. The higher the trust score of a sensor, the more trustworthy the sensor is. Based on the trust scores, we aggregate the probabilistic time series. Instead of treating the sensors and their data equally, we apply a weighted combination of the probabilistic time series based on the trust scores. Consequently, the sensors with high trust scores contribute more to the aggregated probabilistic time series and vice versa. The Aggregating component will be described in detail in Section 3.

Guiding. Even with multiple sensors to mitigate noises, poor data quality cannot be avoided. We employ expert knowledge from human users to improve the quality of aggregated data. Since the amount of noises might be far overwhelming for a user, techniques for minimizing user effort become necessary. This component develops different guiding strategies for effort minimization. Since user input is not known in advance, we estimate the overall accuracy of participatory by probabilistic uncertainty and rely on this notion to select the most beneficial readings for user validation. The more beneficial a data is, the more effort is likely to be spared. The Guiding component will be described in detail in Section 4.

\section{AGGREGATING PARTICIPATORY DATA}

In practice, a community of sensors contains thousands of sensors, which makes it extremely difficult to deal with if we take into account the time series from each sensor separately. As a result, we need to combine the time series emitted from the sensors. Moreover, by combing the data from the sensors, we can leverage the wisdom of the crowd to obtain a higher accuracy for the combined data. One naive approach is to combine the probabilistic time series from the sensors without considering the reliability of the sensors. However, as the quality of the sensors are not the same, this approach misses an important information. To this end, we propose a method to combine probabilistic time series based on the trust scores.

Our method is based on an observation that there is an interdependence between the reliability of the sensors and their data. The quality of the data depends on the sensor that produces them while the reliability of sensors depends on the quality of the data they produce. As a result, we employ a trust model where the trustworthiness of the sensors and their data are measured concurrently and explicitly. More precisely, each sensor is associated with a trust score, which shows its accuracy. The data of the sensor is also assigned a trust score, which represents its probability of correctness. These trust scores provide us a meaningful way to combine probabilistic time series from multiple sensors as well as user input.

The Aggregate component takes as input a set of probabilistic time series $p \mathcal{D}=\left\{p S_{1}, \ldots, p S_{n}\right\}$, which are the results of the Computing Likelihoods component, and user input $e($.$) . It returns an ag-$ gregated time series $G=\left\langle p_{1}\left(G_{1}\right), \ldots, p_{m}\left(G_{m}\right)\right\rangle$ where $p_{j}\left(G_{j}\right)$ is the aggregated probability density function. The aggregated probability density function $p_{j}\left(G_{j}\right)$ at timestamp $j$ is computed from the set of probability density functions $\Omega_{j}=\left\{p_{j}\left(R_{j}^{1}\right), \ldots, p_{j}\left(R_{j}^{n}\right), e_{j}\left(R_{j}\right)\right\}$ 


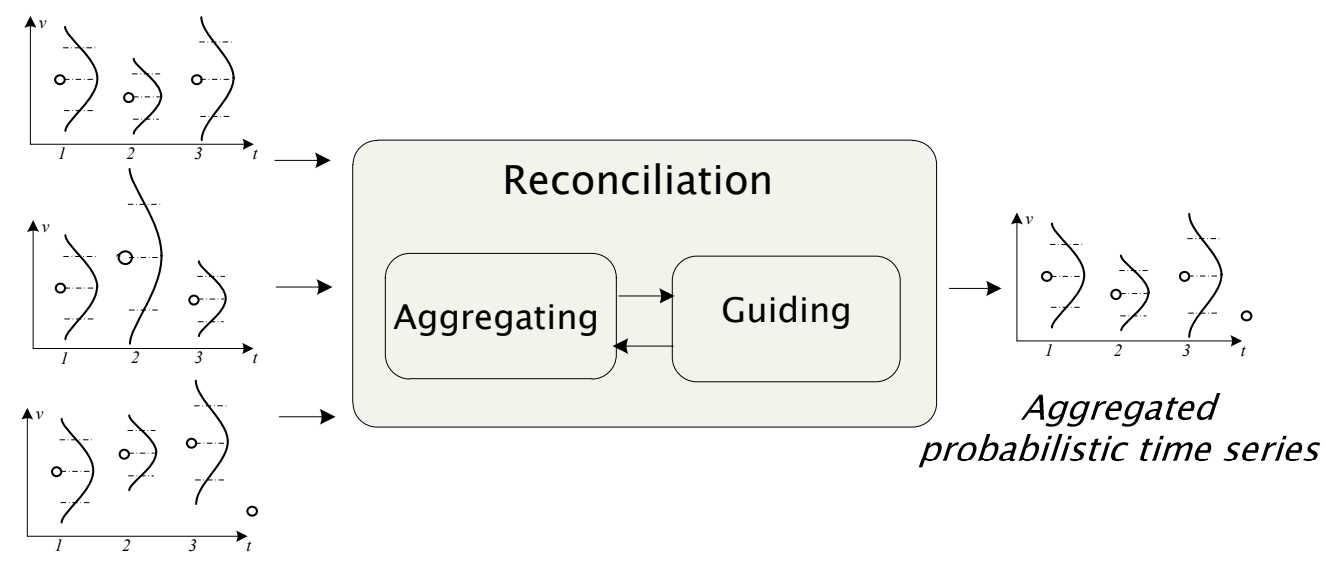

Probabilistic time series

Figure 1: Architecture of the framework.

provided by the sensors at timestamp $j$. In the following, we first discuss our approach to compute the trust scores of the sensors and their data. Then, we explain how to aggregate the probabilistic time series based on the trust scores. Finally, we show how to incorporate user input whenever a new validation of readings is provided.

\subsection{Measuring trust score of sensor data}

Given a set of probability density functions of all sensors at timestamp $j: \Omega_{j}=\left\{p_{j}\left(R_{j}^{1}\right), p_{j}\left(R_{j}^{2}\right), \cdots, p_{j}\left(R_{j}^{n}\right)\right\}$, we need to estimate a trust score $\alpha_{j}^{i}$ for each probability density function $p_{j}\left(R_{j}^{i}\right) \in \Omega_{j}$. In order to compute the trust scores, we consider two factors:

- Similarity of distributions: distributions which are similar to each other should have higher trust scores. The reason is that similar distributions reinforce each other which makes them have a higher chance to be correct. For example, if 5 out of 8 distributions are similar, we tend to consider these 5 distributions correct based on majority rule. Although there may be minor difference between them, the differences may come from small errors in transmission, etc., which can be ignored.

- Reliability of sensors: we observe that a reliable sensor tends to provide correct information. As a result, a distribution which comes from a reliable sensor should have a higher trust score.

Numerically, we can estimate the trust score of distribution $p_{t}\left(R_{t}^{i}\right)$ by taking into account the above factors as follows:

$$
\alpha_{t}^{i}=\frac{\sum_{j=1 . . n, j \neq i} \beta_{j} s_{t}^{i, j}}{\sum_{j=1 . . n} \beta_{j}}
$$

where $\beta_{j} \in[0,1]$ is the trust score of sensor $j$ and $s_{t}^{i, j} \in[0,1]$ is the similarity score of two probability density functions $p_{t}\left(R_{t}^{i}\right), p_{t}\left(R_{t}^{j}\right)$. The domain value of $\alpha_{t}^{i}$ is $[0,1]$ where the higher value of $\alpha_{t}^{i}$, the higher chance that the distribution $p_{t}\left(R_{t}^{i}\right)$ can reflect exactly the true value at timestamp $t$. Note that $\alpha_{t}^{i}=1$ means that all the distributions are the same $\left(\forall j \neq i, s_{t}^{i, j}=1\right)$, whereas $\alpha_{t}^{i}=0$ means that all the trust scores of other sensors are zero $\left(\forall j \neq i, \beta_{j}=0\right)$.

Intuitively, Equation (1) models the relationship between distribution $p_{t}\left(R_{t}^{i}\right)$ and its neighbors (the distributions in $\left.\Omega_{t}\right)$. If it is similar to a neighbor distribution which is provided by a reliable sensor, it should receive a high trust score. On the other hand, the trust score of distribution $p_{t}\left(R_{t}^{i}\right)$ should be low if the distribution is significantly different from the neighbors or the trust scores of the neighbor's sensors are low. For example, when the trust score of the neighbor's sensor is 0 , although the two distributions are very similar $s_{t}^{i, j} \approx 1$, the neighbor distribution $p_{t}\left(R_{t}^{j}\right)$ does not contribute to the trust score of distribution $p_{t}\left(R_{t}^{i}\right)$.

In order to compute Equation (1), we need to calculate the similarity between two distributions and the trust scores of the sensors. In the following, we first discuss the method to measure the similarity between the distribution. Then, we describe our approach to compute the trust scores of the sensors.

Similarity of distributions. Regarding the first task, we employ the Kullback-Leibler $(K L)$ divergence. The KL divergence between two probability density functions $p$ and $q$ is measured as follows:

$$
K L(p \| q)=\int p(x) \log \left(\frac{p(x)}{q(x)}\right)
$$

The divergence value between two distributions is non-negative but it is asymmetric. As a result, to use it as a measure, we employ the following symmetric variation and define it as the dissimilarity between two distributions:

$$
\operatorname{dist}(p, q)=K L(p \| q)+K L(q \| p)
$$

However, as Equation 4 takes the similarity of the distributions as input, we need to calculate the similarity between two distribution from the KL divergence. To this end, consider a set of distributions at timestamp $t \Omega_{t}=\left\{p_{t}\left(R_{t}^{1}\right), p_{t}\left(R_{t}^{2}\right), \cdots, p_{t}\left(R_{t}^{n}\right)\right\}$ provided by the sensors, the similarity score between two distributions can 
be computed as follows:

$$
s_{t}^{i, j}=1-\frac{\operatorname{dist}\left(p_{t}\left(R_{t}^{i}\right), p_{t}\left(R_{t}^{j}\right)\right)}{\operatorname{dist}_{\Omega_{t}}}
$$

where dist $_{\Omega_{t}}$ is the diameter of the distributions in $\Omega_{t}$. The similarity score between the distributions takes a value in $[0,1]$ where the higher the similarity score, the more similar between the distributions. Note that $s_{t}^{i, j}=1$ means the two distributions are the same, where $s_{t}^{i, j}=0$ only means the two distributions are the least similar pair in the set.

Estimating trust scores of sensors. We observe that a sensor that provides more correct data tends to be more reliable. Based on this observation, we can compute the trust scores of the sensors based on the trust scores of the data they provide. As a result, the trust score of a sensor can be calculated as follows:

$$
\beta_{j}=\frac{\sum_{k=1}^{m} \alpha_{k}^{j}}{m}
$$

Or informally we can say that the trust score of a sensor is measured by the average of the trust scores of all the distributions it provides. The domain of $\beta_{j}$ is $[0,1]$. A high value of $\beta_{j}$ represents the high reliability of sensor $j$. Note that $\beta_{j}=1$ means that the sensor provides all distributions with trust scores equal to 1 , whereas $\beta_{j}=0$ means that the sensor does not provide any distribution with positive trust score.

\subsection{Eliciting user input and inferring consequences}

We treat user input as a first-class citizen. Upon the user input of the $z$-th iteration of the validation process for a reading $R_{t}$, we elicit the validation function $e^{z}($.$) as follows: \forall t^{\prime}=1 . . m, t^{\prime} \neq t$, $e^{z}\left(R_{t^{\prime}}\right)=e^{z-1}\left(R_{t^{\prime}}\right)$ and $e^{z}\left(R_{t}\right) \neq \ominus$.

Now we can estimate the trust score of distribution $p_{t}\left(R_{t}^{i}\right)$ from user input directly by computing the similarity between sensing readings and expert readings:

$$
\alpha_{t}^{i}=1-\frac{\operatorname{dist}\left(p_{t}\left(R_{t}^{i}\right), e_{t}^{z}\left(R_{t}\right)\right)}{\operatorname{dist}_{\Omega_{t}}}
$$

Algorithm. We observe that there is an inter-dependence between Eq. 1 (or Eq. 5) and Eq. 4. More specifically, in order to compute the trust score of a sensor $\beta_{j}$, we need to know the trust scores $\alpha_{i}$ of the distributions it provides. Nevertheless, estimating the trust score of a distribution $\alpha_{i}$ requires knowing the trust score of the sensors. There is a mutually reinforcing relationship between the sensor and the data it provides. As a result, we leverage the relationship between them via an iterative algorithm that maintains and updates the trust scores of the sensors and the distributions. We discuss the detail of the iterative algorithm in Alg. 1.

Alg. 1 takes as input a set of probabilistic time series $p \mathcal{D}=$ $\left\{p S_{1}, p S_{2}, \cdots, p S_{n}\right\}$, user input $e^{z}($.$) and returns a set of trust scores$ for the data and the sensors. We first initialize the trust scores of the sensors to 0.5 in Line 1 . The algorithm iterates between two phases: estimating the trust scores of the sensors and the data. In order to estimate the trust score $\alpha_{l}^{i, q}$ of the data at iteration $q$, we use the trust scores of the sensors $\beta_{j}^{q-1}$ which are estimated in the previous

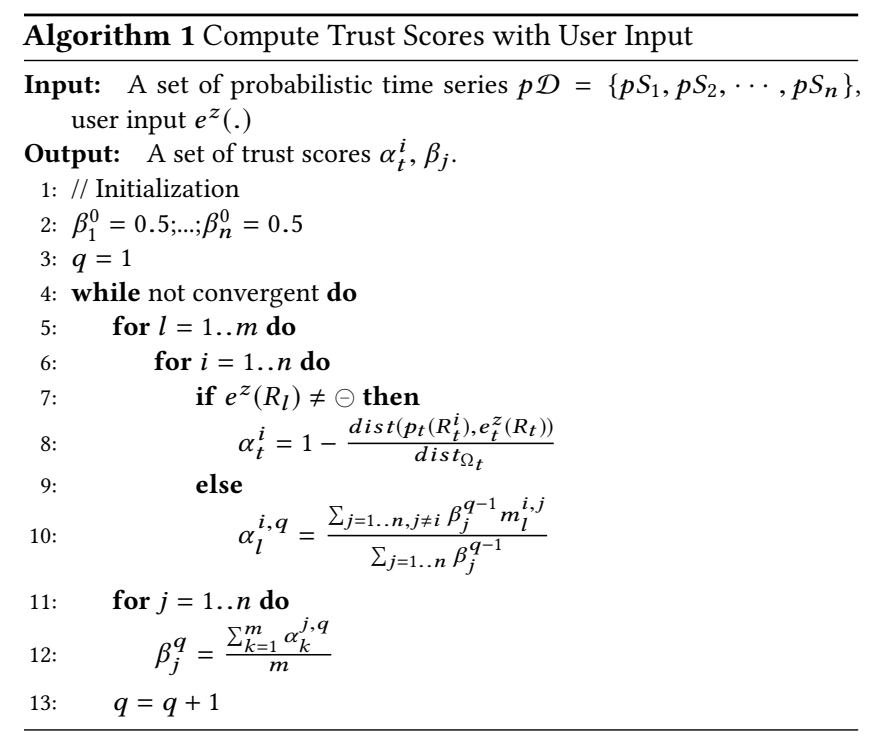

iteration (Line 5-7). Then, in the second phase, we use the trust scores of the data $\alpha_{l}^{i, q}$ to estimate the trust scores of the sensors $\beta_{j}^{q}$ in this iteration (Line 7-10). If the reading is already validated, we update the trust scores according to Eq. 5, and to Eq. 1 otherwise. We continue the iteration until the termination condition $\Delta$ is satisfied. Note that we can reverse the computation by estimating the trust scores of the sensors $\beta_{j}^{q}$ before estimating the trust scores of the data $\alpha_{i}^{q}$. However, this requires an initialization of the trust scores for all the distributions, which is not feasible if the time series is long. Regarding the termination condition $\Delta$, in most of the cases, we want to stop the algorithm when the changes of the trust scores between two iterations are negligible.

\subsection{Deciding the aggregation result}

After the previous step, we have acquired a set of trust scores for the distributions and the sensors. Given the distributions at a timestamp $t$, we need to generate an aggregated distribution that can both represent the distributions and the reliability of the sensors. To this end, we first need to model the relationship between the aggregated distribution and the distributions at timestamp $t$. Based on this relationship and the trust scores, we can generate the aggregated distribution effectively.

Relationship between aggregated and constituent random variables. Since the aggregated distribution at timestamp $t$ is generated from the distributions at the same timestamp, the random variable $G_{t}$ which models the aggregated distribution should be computed from the random variables $R_{t}^{i}$ of the distributions. Another observation is that the contribution of the random variables $R_{t}^{i}$ to $G_{t}$ is different. Intuitively, a distribution which has a higher trust score contributes more to the aggregated distribution. It comes from the fact that the distribution with the higher trust score can express the true value at timestamp $t$ better.

Based on these observations, we can model the relationship between the aggregated and constituent random variables as a 
weighted sum as follows:

$$
G_{t}=\frac{\sum_{i=1}^{n} \alpha_{i} R_{t}^{i}}{\sum_{i=1}^{n} \alpha_{i}}
$$

where $e_{t}^{z}\left(R_{t}\right)=\ominus$.

Decide aggregated probability density functions. Given the random variables of the distributions at timestamp $t$, we can compute the aggregated random variable at the same timestamp based on Eq. 6. Let $M_{R^{i}}$ (.) be the moment-generating function[10] of the random variable $R^{i}$. Since $G$ is the linear combination of independent random variables $R^{1}, R^{2}, \ldots, R^{n}$, its moment-generating function is as follows:

$$
M_{G}(x)=\prod_{i=1}^{n} M_{R^{i}}\left(\alpha_{i} x\right)
$$

However, recall that in our GARCH model, the random variable $R^{i}$ at timestamp $t$ models a normal distribution $\mathcal{N}\left(\hat{r}, \hat{\sigma}^{2}\right)$. Since $R^{i}$ is a normal distribution $\mathcal{N}\left(\hat{r}_{i}, \hat{\sigma}_{i}^{2}\right)$, its moment-generation function $M_{R^{i}}$ is as follows:

$$
M_{R^{i}}(x)=\exp \left(\hat{r}_{i} x+\frac{\hat{\sigma}_{i}^{2} x^{2}}{2}\right)
$$

Therefore, we have

$$
\begin{aligned}
M_{G}(x)=\prod_{i=1}^{n} \exp \left(\hat{r}_{i} \alpha_{i} x\right. & \left.+\frac{\left(\hat{\sigma}_{i}^{2}\right)^{2} \alpha_{i}^{2} x^{2}}{2}\right) \\
& =\exp \left(x\left(\sum_{i=1}^{n} \alpha_{i} \hat{r}_{i}\right)+\frac{x^{2}}{2}\left(\sum_{i=1}^{n} \alpha_{i} \hat{\sigma}_{i}^{2}\right)\right)
\end{aligned}
$$

However, this equation has the same structure as the momentgenerating function for a normal random variable $\mathcal{N}\left(\sum_{i=1}^{n} \alpha_{i} \hat{r}_{i}\right.$, $\left.\sum_{i=1}^{n} \alpha_{i} \hat{\sigma}_{i}^{2}\right)$. From the uniqueness property of moment generating functions, $G$ must be a normal random variable which follows the following normal distribution $\mathcal{N}\left(\sum_{i=1}^{n} \alpha_{i} \hat{r}_{i}, \sum_{i=1}^{n} \alpha_{i} \hat{\sigma}_{i}^{2}\right)$.

Put it altogether with user input. As a result, from the distributions and the trust scores generated, we are able to generate an aggregated distribution at timestamp $t$.

$$
\left.p_{t}\left(G_{t}\right)=\mathcal{N}\left(\sum_{i=1}^{n} \alpha_{i} \hat{r}_{i, t}, \sum_{i=1}^{n} \alpha_{i} \hat{\sigma}_{i, t}^{2}\right)\right)
$$

Applying this approach for other timestamps, we are able to generate an aggregated probabilistic time series $G=\left\langle p_{1}\left(G_{1}\right), \ldots, p_{t}\left(G_{t}\right)\right\rangle$ from the data collected from the sensors.

If we treat user input as a hard constraint, we can decide the probabilistic aggregation for any reading $R_{t}$ that has been validated $\left(e_{t}^{z}\left(R_{t}\right) \neq \ominus\right)$ as:

$$
p_{t}\left(G_{t}\right)=e_{t}\left(R_{t}\right)
$$

otherwise we can use Eq. 7 for all readings.

\section{GUIDING USER IN VALIDATION PROCESS}

Having discussed a model and techniques for (i) inference based on user input and (ii) instantiation of an aggregated probabilistic time series, we now turn to strategies to guide a user in the validation. This section first defines a measure of uncertainty for a participating sensoring data (Section 4.1). Subsequently, we introduce an approach to guide the selection of readings for validation (Section 4.2), before compiling a thorough validation procedure (Section 4.3).

\subsection{Uncertainty of a Participatory Sensing Data}

The model of a participatory sensing data, as constructed above, enables us to quantify the uncertainty related to trust assessment in order to guide a user in the validation process. Let $p \mathcal{D}$ be a participatory sensing data. Recall that we have defined the likelihood of each reading, i.e., $p_{t}\left(G_{t}\right)$. Then, the overall uncertainty of a participatory sensing data is computed by the Shannon entropy [45] over a set of readings as random variables:

$$
H_{\mathcal{R}}(p \mathcal{D})=\sum_{R_{t} \in \mathcal{R}} H\left(p_{t}\left(G_{t}\right)\right)
$$

where $H\left(p_{t}\left(G_{t}\right)\right)=-\int_{0}^{1} p_{t}\left(G_{t}=x\right) \log p_{t}\left(G_{t}=x\right) d x$.

\subsection{Uncertainty-driven User Guidance}

Our approach to guide the selection of readings for validation aims at the maximal reduction in uncertainty. It exploits the benefit of validating a single reading using the notion of information gain from information theory [44].

To capture the impact of user input on a reading $R_{t}$, we define a conditional variant of the entropy measure introduced earlier. Informally, it measures the expected entropy of the participatory sensing data under specific validation input:

$$
H_{\mathcal{R}}\left(p \mathcal{D} \mid R_{t}\right)=\sum_{x \in \Omega} p_{t}\left(G_{t}=x\right) \times H_{\mathcal{R}}(p \mathcal{D} \cup x)
$$

where $p \mathcal{D} \cup x$ is constructed by the aggregating component with $e^{\prime}\left(R_{t}\right)=x$ and $e^{\prime}\left(R_{t^{\prime}}\right)=e\left(R_{t^{\prime}}\right)$ for $t^{\prime}=1 . . m, t^{\prime} \neq t$.

To take a decision on which reading to select, we assess the expected difference in uncertainty before and after incorporating input for a reading. The respective change in entropy is the information gain that quantifies the potential benefit of knowing the true value of an unknown variable [44]:

$$
I G_{\mathcal{R}}\left(R_{t}\right)=H_{\mathcal{R}}(p \mathcal{D})-H_{\mathcal{R}}\left(p \mathcal{D} \mid R_{t}\right) .
$$

The information gain can guide the selection of a reading: we chose the one that is expected to maximally reduce the uncertainty of the participatory sensing data. This is formalized by a selection function for uncertainty-driven user guidance:

$$
\text { select }_{\mathcal{R}}(p \mathcal{D})=\underset{R_{t} \in \mathcal{R}}{\operatorname{argmax}} I G_{\mathcal{R}}\left(R_{t}\right)
$$

While this formulation is sound for ranking a whole set of readings, validated readings will be ranked last (since the uncertainty does not change before and after validating them the second time).

\subsection{Validation Procedure}

Combining the model and inference mechanism introduced in Section 3 with the above strategy to guide a user, we present our complete validation process for participatory sensing data in Alg. 2. As long as the validation goal is not reached and the user effort budget has not been exhausted (line 5), selection of the reading for which user input shall be sought is done by the uncertainty-driven 
strategy (line 7). The second step (lines 9) elicits user input for the selected reading. The third step (line 11-14) incorporates the user input. That is, we update the validation function $e_{i+1}$; infer the implications of user input by means of function conclude, which yields a new probabilistic information of participatory sensing data; and decide on the new probabilistic aggregation $g_{i+1}$ capturing which readings are considered credible. Further, the trustworthiness of each sensor is updated (lines 15), which is used in the next iteration to choose between the selection strategies.

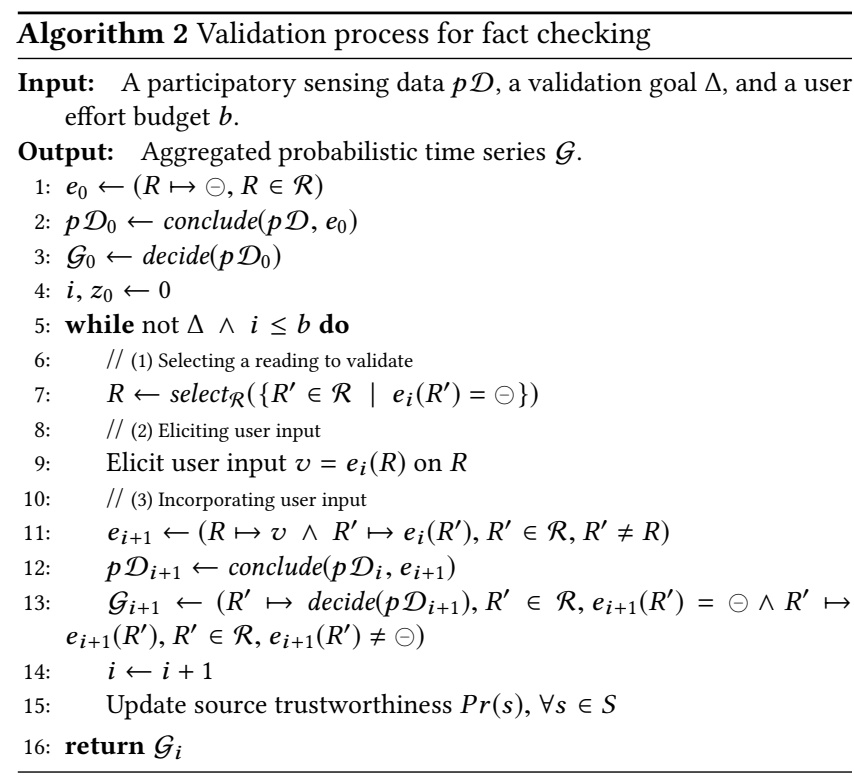

\section{EXPERIMENTAL EVALUATION}

In this section, we evaluate the proposed approach experimentally, using real-world and synthetic datasets. We first discuss the experimental setup (Section 5.1), before turning to an evaluation of the following aspects of our approach:

- The efficiency and effectiveness of computing trust scores (Section 5.2).

- The effectiveness of aggregating probabilistic data (Section 5.3).

- The efficiency, efficacy and effectiveness of user guidance (Section 5.4).

Since different baselines are not applicable for all experimental setting, we defer the description of baselines to each evaluation.

\subsection{Experimental Setup}

5.1.1 Datasets. In our experiments, we use two types of data: real-world data and synthetic data. While the real-world data shows us a pragmatic view of the real settings, the synthetic data provides different settings which the real-world dataset cannot cover.

Real-world Data. We relied on two real-world datasets covering different domains where participatory sensing can be applied. (1) Campus: this dataset contains temperature readings collected from a real sensor network deployed on the EPFL university campus. The dataset is built over twenty five days and includes about eighteen thousand samples. It is referred in this paper under the name campus-data. (2) Moving Object: the dataset contains about ten thousand GPS readings collected over five and a half hours from 192 cars in Copenhagen, Denmark. Each log entry contains the time and both the $x-y$ coordinates. In this dataset, which we refer as car-data, only the $\mathrm{x}$-coordinate is used in the experiments.

Table 2: Summary of Datasets

\begin{tabular}{|c|c|c|}
\hline & campus-data & car-data \\
\hline Monitored parameter & Temperature & GPS Position \\
Number of data values & 18031 & 10473 \\
Sensor accuracy & \pm 0.3 deg. C & \pm 10 meters \\
Sampling interval & 2 minutes & $1-2$ seconds \\
\hline
\end{tabular}

An important observation regarding the real-world datasets is that the car-data dataset is smoother than the campus-data one. This is expected as cars always follow a predefined path of roads and highways, which makes the changes in $\mathrm{x}-\mathrm{y}$ coordinates smooth. We summarize the important properties of the real dataset in Table 2.

Synthetic Data. In order to evaluate our proposed method in various scenarios, we generate a synthetic dataset to be able to control the parameters. At each timestamp, we first generate a true distribution which we assume to best model the expected true value for this timestamp. Then, at the same timestamp, we construct a normal distribution for each sensor which is assumed to present the readings collected from the sensor. The normal distribution is constructed from the true distribution by adding a small difference to the mean of the true distribution. By applying this method to all the timestamps, we are able to generate the synthetic dataset.

5.1.2 Evaluation Metrics. In addition to the uncertainty of the participatory data defined in Section 4, we relied on the following measures:

Relative user efforts $\left(E_{i}\right)$ : the number of user-validated readings $i$ relative to the number of all readings $|\mathcal{R}|$, i.e., $E=i /|\mathcal{R}|$.

Accuracy $\left(A_{i}\right)$ : the correctness of the probabilistic aggregation at each validation step. Let $p^{*}$ be the correct distribution of reading values. Then, the accuracy of the probabilistic aggregation $\mathcal{G}_{i}$ at the $i$-th validation step is

$$
A_{i}=\frac{\sum_{R_{t} \in \mathcal{R}} \operatorname{dist}\left(p_{i}\left(G_{t}\right), p^{*}\left(G_{t}\right)\right)}{|\mathcal{R}|} .
$$

5.1.3 Experimental environment. Experimental results have been obtained on an Intel Core i7 system (3.4GHz, 12GB RAM). All experiments ran until the actual termination of the validation process.

\subsection{Evaluations on computing trust}

In the following experiments, we want to analyze the Trust assessment component in different aspects, including computation time, convergence, effects of outliers, and effects of distance.

Computation time. In practice, data from the sensors are used in various applications which require good response. As a result, the computation time to calculate the trust scores is critical to the performance of these applications. In this experiment, we want to analyze the running time of our algorithm with different settings. More specifically, we vary the number of sensors from 10 to 30 and 
the length of the time series from 100 to 400 . The experimental result is illustrated in Table 3.

Table 3: Running time of Algorithm 1 (s)

\begin{tabular}{|c|c|c|c|c|}
\hline \multirow{2}{*}{ \#Sensors } & \multicolumn{4}{|c|}{ \#Timestamps } \\
\cline { 2 - 5 } & 100 & 200 & 300 & 400 \\
\hline 10 & 1.85 & 3.67 & 5.50 & 7.40 \\
20 & 2.13 & 3.71 & 5.61 & 9.53 \\
30 & 6.11 & 12.32 & 18.33 & 24.58 \\
\hline
\end{tabular}

A significant observation is that the running time increases linearly with the increase in the length of the time series. For instance, when the number of timestamps triples from 100 to 300, the computation time also increases three times despite of the change in the number of sensors. On the other hand, when the number of sensors increases, the running time does not increase linearly. The reason is that we need to measure the similarity between every pair of distributions provided by the sensors. As the number of sensors increases, the number of pairs increases significantly.

Convergence. In this experiment, we want to find out how quickly Alg. 1 converges and under which condition. To this end, we run Alg. 1 with the synthetic dataset to test the algorithm in different settings.

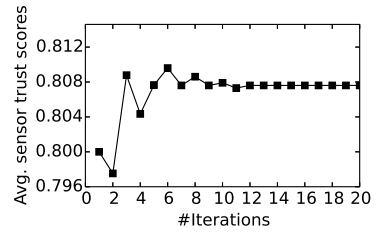

Figure 2: Convergence of Alg. 1

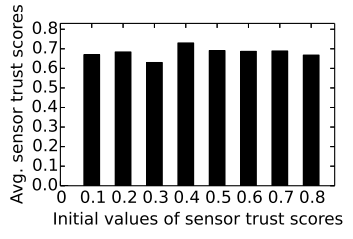

Figure 3: Effects of initialization
We first measure the number of iterations the algorithm requires before it converges to demonstrate the quick convergence rate. The convergence rate is reflected through the average value of the trust scores of the sensors. The experimental result is shown in Fig. 2. The $\mathrm{X}$-axis is the number of iterations. The Y-axis is the average trust score. A key observation is that the algorithm converges very fast. After 10 iterations, the average trust score of all the sensors remains nearly the same. As a result, this shows that our algorithm can be used for applications that require good performance as it can finish after a few iterations.

Second, we measure the convergence rate with different initializations to show that the algorithm is robust. More specifically, we vary the initial values of $\beta$ in Alg. 1 and measure the final average trust score for different settings. The algorithm is set to terminate when the change in the average trust score of all the sensors between two iterations are less than $10^{-8}$. The result is illustrated in Fig. 3. The $\mathrm{X}$-axis is the initial value and the $\mathrm{Y}$-axis is the average trust score. It can clearly be seen that Alg. 1 does not depend on the initial values of $\beta$. As we change the initial values $\beta$, the algorithm still converges to the same result.
Effect of outliers. In this experiment, we want to analyze the accuracy of the proposed algorithm in computing the trust scores. To this end, we generate some outliers (sensors which data are completely different from the rest) among the sensors. In reality, some sensors may produce data that are completely different from other sensors. The reason is that their communication channels are blocked by buildings, walls or their processing units have severe problems. In these cases, applications or methods that use the data provided by these spammer sensors may produce incorrect results. By increasing the percentage of outliers in the network of sensors from 10 to $50 \%$, we want to verify our hypothesis that the outliers receive low trust scores. The experimental results is shown in Fig. 4. The $\mathrm{X}$-axis and the $\mathrm{Y}$-axis represents the percentage of outliers and the trust score, respectively.

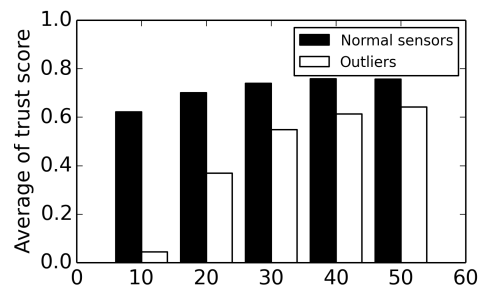

Figure 4: Accuracy of the algorithm

A key observation is that the outliers and normal sensors can be clearly separated. At each value of the percentage of outliers, the normal sensors which always have high trust scores while the outliers always have lower trust scores. This distinction shows that our algorithm is able to distinguish between normal sensors and outliers. Another interesting finding is that the trust scores of normal sensors are still higher than the outliers even the percentage of outliers is high (40 or 50\%).

Effects of distance between distributions. In the previous experiment, we have demonstrated the accuracy of the algorithm when calculating the trust scores of the sensors. In this experiment, we want to analyze the accuracy of the algorithms regarding the trust scores of the data i.e., the distributions. Recall that in order to construct the synthetic dataset, we first generate a true distribution at each timestamp. Based on the true distribution, we generate the distributions of the sensors by adding small differences to the mean of the true distribution. Now after generating the synthetic dataset, we rank the distributions at a timestamp by how close it is to the true distribution. The closer a distribution to the true distribution, the higher rank it receives. After running Alg. 1, we calculate the rank of the distribution again where the distribution with the higher trust score has the higher rank.

The experimental result is shown in Fig. 5 , in which we vary the distance between the distributions $(0.02$ and 0.2$)$. The $\mathrm{X}$-axis is the correct ranking (w.r.t true distribution), while the Y-axis is the estimated ranking (w.r.t trust score). Each point in the two plots of Fig. 5 represents a distribution located by the ranking of the distribution before and after running the algorithm. As a result, there may be many distributions that could have the same true rank and estimated rank. We illustrate this factor by the size of the point: 

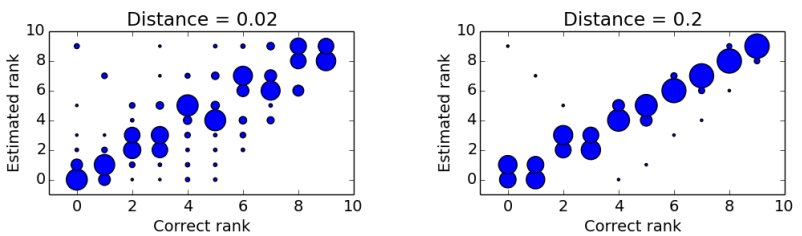

Figure 5: Effect of distance between distributions on the accuracy of computing trust

the larger the point is, the more distributions that have the same true rank and estimated rank at that point.

Intuitively, the closer the point to the diagonal, the better since it shows that the true rank of a distribution measured before and the estimated rank measured after running the algorithm are nearly the same. This means the algorithm is able to assess the trust scores of the distributions correctly. A key observation from Fig. 5 is that the number of points located near the diagonal is significantly high. This shows that our algorithm is able to estimate the ranks of the distributions correctly, i.e., the trust scores of the distributions are also correct. We also observe that by increasing the distance between the distributions, the number of points located near the diagonal increase. Therefore, it shows that the accuracy of our algorithm increases if the differences between the distributions are high.

\subsection{Evaluations on aggregating distributions}

We now analyze the performance of our aggregating component via the following experiments.

Effects of heterogeneity level. As mentioned in previous section, quality of the sensors are different from one sensor to another. As a result, the data generated by the sensors are also different i.e., the heterogeneity level among the sensors are high. In this experiment, we want to analyze the effect of heterogeneity level to the performance of our proposed method. More specifically, we vary the difference between the means of the distributions and the mean of the true distribution from 1 to 20 . The experimental result is shown in Fig. 6. The $\mathrm{X}$-axis is the relative difference between the two means, whereas the Y-axis is the distance against the true distribution.

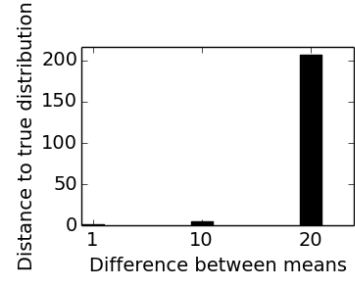

Figure 6: Distance to true distribution w.r.t percentage of change in mean

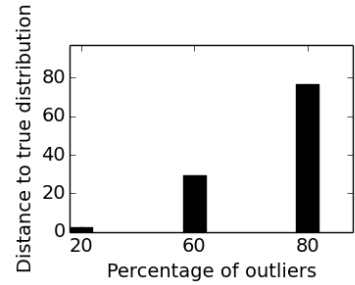

Figure 7: Distance to true distribution w.r.t percentage of outliers
An interesting observation is that the distance to the true distribution increases as the difference increases. This can be explained as follows: since we increase the difference between the means of the distributions of the sensors and the mean of the true distribution, the distributions of the sensors become deviating from the true distribution. As a result, the more different between the distributions and the true distribution, the more different between the aggregated distribution and the true distribution. Therefore, the distance between the true distribution and the aggregated distribution increases as the difference increases.

Effects of outliers. In this experiment, we want to find out the effects of the outlier sensors to the performance of our Aggregating component. We measure the accuracy of our approach by comparing the distance of the aggregated distributions to the true distributions. The experimental result is illustrated in Fig. 7. The $\mathrm{X}$-axis is the percentage of outliers among all sensors, while the $\mathrm{Y}$-axis is the distance against the true distributions.

An interesting observation is that the distance against the true distribution increases as the number of outliers increases. This phenomenon is expected and can be explained as follows: as the number of outliers increases, they are able to pollute the data provided by correct sensors. However, as we expect correct sensors to provide distributions that are similar to the true distribution, the data provided by outliers will make the aggregated distribution different from the true distribution. Therefore, the more outliers, the more different between the aggregated distribution and the true distribution.

\subsection{Evaluations on guiding users}

We now evaluate the performance of our guiding component. User input is simualted by means of ground-truth of the datasets themselves.

Computation time. Being based on user interactions, our approach relies on a good runtime performance. Therefore, a first experiment measures the response time, denoted by $\Delta t$, of our approach during one iteration of Alg. 2, i.e., the waiting time of a user after validating one reading. This includes the time to infer implications of user input and to select the next best reading to validate.

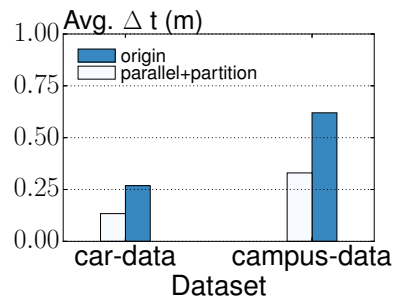

Figure 8: Time vs. dataset

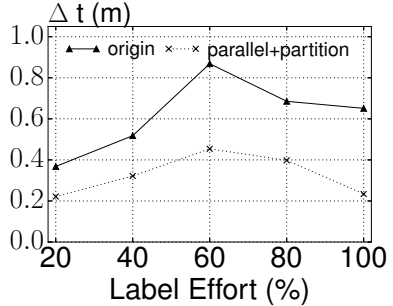

Figure 9: Time vs. effort
Fig. 8 shows the observed response time, averaged over 10 runs, when using the plain algorithm (origin) and with the computational optimisations (parallel+partition). With larger dataset size (car-data to campus-data), the response time increases. However, with computational optimisations, the average response time stays below 
half a minute, which enables good user interactions. Fig. 9 further illustrates for the largest dataset, campus-data, how the response time evolves during the validation process when averaging the response time over equal bins of user effort. The time peaks between $40 \%$ and $60 \%$ of user effort, since at these levels, user input enables the most conclusions on the true value of readings.

Relation between uncertainty and precision. We verify the underlying assumption of our approach to user guidance, i.e., that the uncertainty of a participatory data, see Section 4, is correlated with the accuracy of the probabilistic aggregation. In this experiment, the uncertainty-driven guidance strategy was applied to all datasets (100 runs each), until accuracy reaches 1.0. Fig. 10 depicts the results in terms of accuracy and normalized uncertainty (i.e., uncertainty divided by the maximum value of the run). There is a strong correlation between both measures (Pearson's correlation coefficient is $-0.8523)$. Hence, uncertainty is indeed a truthful indicator of the result correctness.

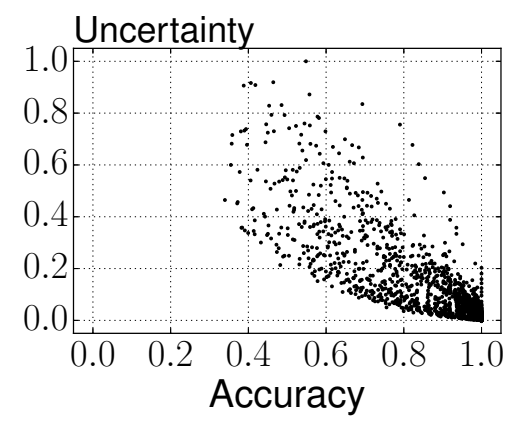

Figure 10: Uncertainty vs. accuracy

Guidance strategies. Turning to the guidance strategies, we mimic the user by means of the ground-truth, until precision reaches 1.0. We compare the proposed approach (info) with two methods: random, which selects a reading randomly for validation; and semantic, which selects the reading that is most 'problematic' in terms of the entropy of its probability. Intuitively, this semantic method outperforms random selection since it targets readings that are on the edge of being considered trusted and thus the major sources of model uncertainty.
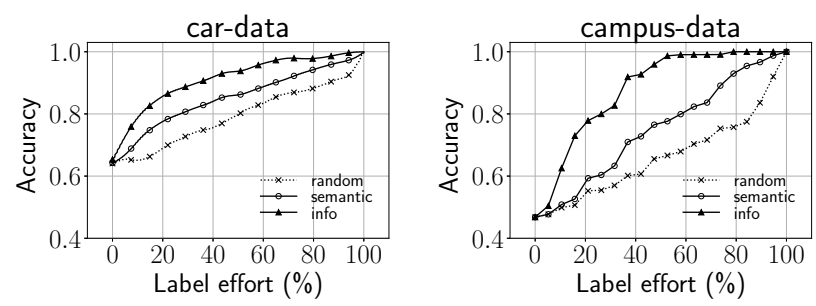

Figure 11: Effectiveness of guiding

Fig. 11 shows the results for the campus and car datasets. Our approach (info) clearly outperforms random selection and the baseline method. For example, using the campus dataset, our approach leads to an accuracy above 0.9 with input on only $31 \%$ of the readings, whereas the other methods require validation of at least $84 \%$ to reach the same level of accuracy.

\section{RELATED WORK}

In the following, we review the work in participatory sensing and user guidance that is close to our research.

Participatory sensing. In recent years, there has been an increased interest on participatory sensing due to the increasing number of personal sensors such as smartphones, GPS devices, and cameras $[12,30,32,51,59,60]$. Participatory sensing campaigns often recruit participants to provide measurements on a particular region or a population. The examples of these campaigns include monitoring traffic [63], measuring the level of pollution [35], or documenting quality of roads[42]. As the quality of the sensors are unknown beforehand, many methods to assess the quality of the sensors and find the correct data (truth finding) have been proposed. These truth finding methods aim to find the correct data from the readings provided by the sensors. An approach to tackle truth finding in participatory sensing is proposed in [52] where the authors employ the Expectation Maximization algorithm to estimate the correct data. Although our proposed approach also estimates the quality of the sensors, our approach is different from [52] as we focus on generating probabilistic databases instead of finding a single correct value for the data (the nature of sensor data is stochastic itself). It is noteworthy that participatory sensing is quite similar to crowdsourcing, in which data are provided by multiple workers. Each worker also has a "trust" score that reflects the quality of his answers. In this sense, we could apply answer aggregation techniques [21] from the crowdsourcing setting to our setting. However, we should be careful about the main difference of input data between the two settings. That is, data provided by workers are multiple-choice answers; whereas, data provided by sensors are real values $[1,9,13,14,16-18,21,22,31,46]$.

User guidance. Guiding users or experts has been studied in data integration, data repair, crowdsourcing, and recommender systems $[33,34,37,55]$. Following this line, we rely on models from the fields of Decision Theory and Active Learning [43, 44]. Despite the similarities in the applied models, our approach differs from the aforementioned ones in several ways. First, unlike existing work that focuses on structured data that is deterministic and traceable, we cope with sensor data that is unreliable and non-deterministic. Second, the benefit of user input depends on the application domain and, unlike the above works, our approach is tailored to the specific characteristics of sensor data. Finally, we do not require any training data for a user to begin the validation process and we incrementally incorporate user input without devising a model from scratch upon receiving new inputs.

\section{CONCLUSIONS}

In this paper, we build a systematic model to manage and validate uncertain data from participatory sensors. We define the notion of probability distribution for a time series as the backbone of our approach. Most importantly, our probabilistic model allows the 
capture of the dynamic and uncertain nature of participatory sensing data. Our approach involves two steps. In the first step, we unify probabilistic data from multiple sensors by using trust-based aggregation. In the third step, we provide a guiding mechanism for user to validate the probabilistic data. We presented a comprehensive experimental evaluation of each of the steps, indicating that the approach is applicable for real-world datasets and allows for effective and efficient management and validation of participatory sensing data.

\section{REFERENCES}

[1] Duong Tuan Anh, Vo Hoang Tam, and Nguyen Quoc Viet Hung. 2006. Generating complete university course timetables by using local search methods. In RIVF. 67-74.

[2] Hongxu Chen, Hongzhi Yin, Weiqing Wang, Hao Wang, Quoc Viet Hung Nguyen, and Xue Li. 2018. PME: Projected Metric Embedding on Heterogeneous Networks for Link Prediction. In KDD. 1177-1186.

[3] Reynold Cheng, Dmitri V Kalashnikov, and Sunil Prabhakar. 2003. Evaluating probabilistic queries over imprecise data. In SIGMOD. 551-562.

[4] Graham Cormode and Minos Garofalakis. 2007. Sketching probabilistic data streams. In SIGMOD. 281-292.

[5] Nilesh Dalvi and Dan Suciu. 2007. Efficient query evaluation on probabilistic databases. FVLDB (2007), 523-544.

[6] Manh Truong Dang, Anh Vu Luong, Tuyet-Trinh Vu, Quoc Viet Hung Nguyen, Tien Thanh Nguyen, and Bela Stantic. 2018. An Ensemble System with Random Projection and Dynamic Ensemble Selection. In ACIIDS. 576-586.

[7] Xiaolong Deng, Yingtong Dou, Tiejun Lv, and Quoc Viet Hung Nguyen. 2017. A Novel Centrality Cascading Based Edge Parameter Evaluation Method for Robust Influence Maximization. IEEE Access 5 (2017), 22119-22131.

[8] Chi Thang Duong, Quoc Viet Hung Nguyen, Sen Wang, and Bela Stantic. 2017. Provenance-Based Rumor Detection. In ADC. 125-137.

[9] Avigdor Gal, Michael Katz, Tomer Sagi, Matthias Weidlich, Karl Aberer, Nguyen Quoc Viet Hung, Zoltán Miklós, Eliezer Levy, and Victor Shafran. 2013. Completeness and Ambiguity of Schema Cover. In CoopIS. 241-258.

[10] Charles Miller Grinstead and James Laurie Snell. 1998. Introduction to probability. American Mathematical Soc.

[11] Ming Hua, Jian Pei, Wenjie Zhang, and Xuemin Lin. 2008. Ranking queries on uncertain data: a probabilistic threshold approach. In SIGMOD. 673-686.

[12] Nguyen Quoc Viet Hung and Duong Tuan Anh. 2007. Combining SAX and Piecewise Linear Approximation to improve similarity search on financial time series. In ISITC. 58-62.

[13] Nguyen Quoc Viet Hung and Duong Tuan Anh. 2008. An Improvement of PAA for Dimensionality Reduction in Large Time Series Databases. In PRICAI. 698-707.

[14] Nguyen Quoc Viet Hung and Duong Tuan Anh. 2013. Using motif information to improve anytime time series classification. In SoCPaR. 1-6.

[15] Nguyen Quoc Viet Hung, Chi Thang Duong, Nguyen Thanh Tam, Matthias Weidlich, Karl Aberer, Hongzhi Yin, and Xiaofang Zhou. 2017. Argument discovery via crowdsourcing. VLDB 7. 26, 4 (2017), 511-535.

[16] Nguyen Quoc Viet Hung, Hoyoung Jeung, and Karl Aberer. 2013. An Evaluation of Model-Based Approaches to Sensor Data Compression. TKDE 25, 11 (2013), 2434-2447.

[17] Nguyen Quoc Viet Hung, Xuan Hoai Luong, Zoltán Miklós, Thanh Tho Quan, and Karl Aberer. 2013. Collaborative Schema Matching Reconciliation. In CoopIS. 222-240.

[18] Nguyen Quoc Viet Hung, Xuan Hoai Luong, Zoltán Miklós, Thanh Tho Quan, and Karl Aberer. 2013. An MAS negotiation support tool for schema matching. In AAMAS. 1391-1392.

[19] Nguyen Quoc Viet Hung, Saket Sathe, Chi Thang Duong, and Karl Aberer. 2014 Towards enabling probabilistic databases for participatory sensing. In CollaborateCom. $114-123$.

[20] Nguyen Quoc Viet Hung, Nguyen Thanh Tam, Vinh Tuan Chau, Tri Kurniawan Wijaya, Zoltán Miklós, Karl Aberer, Avigdor Gal, and Matthias Weidlich. 2015. SMART: A tool for analyzing and reconciling schema matching networks. In ICDE. 1488-1491.

[21] Nguyen Quoc Viet Hung, Nguyen Thanh Tam, Ngoc Tran Lam, and Karl Aberer 2013. BATC: a benchmark for aggregation techniques in crowdsourcing. In SIGIR. 1079-1080.

[22] Nguyen Quoc Viet Hung, Nguyen Thanh Tam, Zoltán Miklós, and Karl Aberer. 2013. On Leveraging Crowdsourcing Techniques for Schema Matching Networks. In DASFAA. 139-154.

[23] Nguyen Quoc Viet Hung, Nguyen Thanh Tam, Zoltán Miklós, and Karl Aberer. 2014. Reconciling Schema Matching Networks Through Crowdsourcing. EAI 1, 2 (2014), e2.
[24] Nguyen Quoc Viet Hung, Nguyen Thanh Tam, Zoltán Miklós, Karl Aberer, Avigdor Gal, and Matthias Weidlich. 2014. Pay-as-you-go reconciliation in schema matching networks. In ICDE. 220-231.

[25] Nguyen Quoc Viet Hung, Duong Chi Thang, Nguyen Thanh Tam, Matthias Weidlich, Karl Aberer, Hongzhi Yin, and Xiaofang Zhou. 2017. Answer validation for generic crowdsourcing tasks with minimal efforts. VLDB f. 26, 6 (2017), 855-880.

[26] Nguyen Quoc Viet Hung, Duong Chi Thang, Matthias Weidlich, and Karl Aberer. 2015. ERICA: Expert Guidance in Validating Crowd Answers. In SIGIR. 10371038.

[27] Nguyen Quoc Viet Hung, Do Son Thanh, Nguyen Thanh Tam, and Karl Aberer. 2014. Privacy-Preserving Schema Reuse. In DASFAA. 234-250.

[28] Nguyen Quoc Viet Hung, Do Son Thanh, Nguyen Thanh Tam, and Karl Aberer. 2015. Tag-Based Paper Retrieval: Minimizing User Effort with Diversity Awareness. In DASFAA. 510-528.

[29] Nguyen Quoc Viet Hung, Huynh Huu Viet, Nguyen Thanh Tam, Matthias Weidlich, Hongzhi Yin, and Xiaofang Zhou. 2018. Computing Crowd Consensus with Partial Agreement. TKDE 30, 1 (2018), 1-14.

[30] Nguyen Quoc Viet Hung, Huynh Huu Viet, Nguyen Thanh Tam, Matthias Weidlich, Hongzhi Yin, and Xiaofang Zhou. 2018. Extended: Computing Crowd Consensus with Partial Agreement. In ICDE. 1-2.

[31] Nguyen Quoc Viet Hung, Tri Kurniawan Wijaya, Zoltán Miklós, Karl Aberer, Eliezer Levy, Victor Shafran, Avigdor Gal, and Matthias Weidlich. 2013. Minimizing Human Effort in Reconciling Match Networks. In ER. 212-226.

[32] Nguyen Quoc Viet Hung, Kai Zheng, Matthias Weidlich, Bolong Zheng, Hongzhi Yin, Nguyen Thanh Tam, and Bela Stantic. 2018. What-if Analysis with Conflicting Goals: Recommending Data Ranges for Exploration. In ICDE. 1-12.

[33] Shawn R. Jeffery, Michael J. Franklin, and Alon Y. Halevy. 2008. Pay-as-you-go user feedback for dataspace systems. In SIGMOD. 847-860.

[34] Yaguang Li, Han Su, Ugur Demiryurek, Bolong Zheng, Tieke He, and Cyrus Shahabi. 2017. PaRE: A System for Personalized Route Guidance. In WWW. 637-646.

[35] Min Mun, Sasank Reddy, Katie Shilton, Nathan Yau, Jeff Burke, Deborah Estrin, Mark Hansen, Eric Howard, Ruth West, and Péter Boda. 2009. PEIR, the personal environmental impact report, as a platform for participatory sensing systems research. In MobiSys. 55-68.

[36] Dan Olteanu, Jiewen Huang, and Christoph Koch. 2009. Sprout: Lazy vs. eager query plans for tuple-independent probabilistic databases. In ICDE. 640-651.

[37] Aditya G Parameswaran, Hector Garcia-Molina, Hyunjung Park, Neoklis Polyzotis, Aditya Ramesh, and Jennifer Widom. 2012. Crowdscreen: Algorithms for filtering data with humans. In SIGMOD. 361-372.

[38] Douglas Alves Peixoto and Nguyen Quoc Viet Hung. 2016. Scalable and Fast Top-k Most Similar Trajectories Search Using MapReduce In-Memory. In ADC. 228-241.

[39] Douglas Alves Peixoto, Han Su, Nguyen Quoc Viet Hung, Bela Stantic, Bolong Zheng, and Xiaofang Zhou. 2018. Concept for Evaluation of Techniques for Trajectory Distance Measures. In MDM. 276-277.

[40] Douglas Alves Peixoto, Xiaofang Zhou, Nguyen Quoc Viet Hung, Dan He, and Bela Stantic. 2018. A System for Spatial-Temporal Trajectory Data Integration and Representation. In DASFAA. 807-812.

[41] Christopher Ré, Julie Letchner, Magdalena Balazinksa, and Dan Suciu. 2008. Event queries on correlated probabilistic streams. In SIGMOD. 715-728.

[42] Sasank Reddy, Katie Shilton, Gleb Denisov, Christian Cenizal, Deborah Estrin, and Mani Srivastava. 2010. Biketastic: sensing and mapping for better biking. In CHI. 1817-1820.

[43] Neil Rubens, Mehdi Elahi, Masashi Sugiyama, and Dain Kaplan. 2015. Active learning in recommender systems. In Recommender systems handbook. 809-846.

[44] Stuart J. Russell and Peter Norvig. 2003. Artificial Intelligence: A Modern Approach. Pearson Education.

[45] Claude E Shannon. 2001. A mathematical theory of communication. ACM SIGMOBILE Mobile Computing and Communications Review (2001), 3-55

[46] Nguyen Thanh Tam, Nguyen Quoc Viet Hung, and Thanh Tho Quan. 2012. A Framework to Combine Multiple Matchers for Pair-Wise Schema Matching. In RIVF. 1-6.

[47] Nguyen Thanh Tam, Nguyen Quoc Viet Hung, Matthias Weidlich, and Karl Aberer. 2015. Result selection and summarization for Web Table search. In ICDE. 231-242.

[48] Nguyen Thanh Tam, Matthias Weidlich, Duong Chi Thang, Hongzhi Yin, and Nguyen Quoc Viet Hung. 2017. Retaining Data from Streams of Social Platforms with Minimal Regret. In IFCAI. 2850-2856.

[49] Yufei Tao, Reynold Cheng, Xiaokui Xiao, Wang Kay Ngai, Ben Kao, and Sunil Prabhakar. 2005. Indexing multi-dimensional uncertain data with arbitrary probability density functions. In VLDB. 922-933.

[50] Duong Chi Thang, Nguyen Thanh Tam, Nguyen Quoc Viet Hung, and Karl Aberer. 2015. An Evaluation of Diversification Techniques. In DEXA. 215-231.

[51] Nguyen Thanh Toan, Phan Thanh Cong, Nguyen Thanh Tam, Nguyen Quoc Viet Hung, and Bela Stantic. 2018. Diversifying Group Recommendation. IEEE Access 6 (2018), 17776-17786. 
[52] Dong Wang, Lance Kaplan, Hieu Le, and Tarek Abdelzaher. 2012. On Truth Discovery in Social Sensing: A Maximum Likelihood Estimation Approach. In IPSN. 233-244.

[53] Weiqing Wang, Hongzhi Yin, Zi Huang, Xiaoshuai Sun, and Nguyen Quoc Viet Hung. 2018. Restricted Boltzmann Machine Based Active Learning for Sparse Recommendation. In DASFAA. 100-115.

[54] Weiqing Wang, Hongzhi Yin, Zi Huang, Qinyong Wang, Xingzhong Du, and Quoc Viet Hung Nguyen. 2018. Streaming Ranking Based Recommender Systems. In SIGIR. 525-534.

[55] Mohamed Yakout, Ahmed K Elmagarmid, Jennifer Neville, Mourad Ouzzani, and Ihab F Ilyas. 2011. Guided data repair. In VLDB. 279-289.

[56] Hongzhi Yin, Hongxu Chen, Xiaoshuai Sun, Hao Wang, Yang Wang, and Quoc Viet Hung Nguyen. 2017. SPTF: A Scalable Probabilistic Tensor Factorization Model for Semantic-Aware Behavior Prediction. In ICDM. 585-594.

[57] Hongzhi Yin, Liang Chen, Weiqing Wang, Xingzhong Du, Nguyen Quoc Viet Hung, and Xiaofang Zhou. 2017. Mobi-SAGE: A Sparse Additive Generative Model for Mobile App Recommendation. In ICDE. 75-78.

[58] Hongzhi Yin, Zhiting Hu, Xiaofang Zhou, Hao Wang, Kai Zheng, Nguyen Quoc Viet Hung, and Shazia Wasim Sadiq. 2016. Discovering interpretable geo-social communities for user behavior prediction. In ICDE. 942-953.

[59] Hongzhi Yin, Nguyen Quoc Viet Hung, Zi Huang, and Xiaofang Zhou. 2018. Joint Event-Partner Recommendation in Event-based Social Networks. In ICDE. 1-12.

[60] Hongzhi Yin, Weiqing Wang, Liang Chen, Xingzhong Du, Quoc Viet Hung Nguyen, and Zi Huang. 2018. Mobi-SAGE-RS: A sparse additive generative model-based mobile application recommender system. KBS 157 (2018), 68-80.

[61] Hongzhi Yin, Xiaofang Zhou, Bin Cui, Hao Wang, Kai Zheng, and Nguyen Quoc Viet Hung. 2016. Adapting to User Interest Drift for POI Recommendation. TKDE 28, 10 (2016), 2566-2581.

[62] Yan Zhao, Shuo Shang, Yu Wang, Bolong Zheng, Quoc Viet Hung Nguyen, and Kai Zheng. 2018. REST: A Reference-based Framework for Spatio-temporal Trajectory Compression. In KDD. 2797-2806.

[63] Pengfei Zhou, Yuanqing Zheng, and Mo Li. 2012. How Long to Wait?: Predicting Bus Arrival Time with Mobile Phone Based Participatory Sensing. In MobiSys. 379-392. 